\title{
Correction to: Unlabeled PCA-shuffling initialization for convolutional neural networks
}

\author{
Jun $\mathrm{Ou}^{1} \cdot$ Yujian $\mathrm{Li}^{1} \cdot$ Chengkai Shen ${ }^{1}$
}

Published online: 25 August 2018

(C) Springer Science+Business Media, LLC, part of Springer Nature 2018

\section{Correction to: Applied Intelligence} https://doi.org/10.1007/s10489-018-1230-2

The original version of this article unfortunately contained a mistake. Errors in the online version as follows:

(1) In the 2 nd row of Column $8(\operatorname{Rel}(\%))$ in Table 5, the number " 54814 " should be changed to " 5.4814 ".

(2) In the 3 nd row of Column $8(\operatorname{Rel}(\%))$ in Table 5 , the number " 106743 " should be changed to " 10.6743 ".

(3) In the 3nd row of Column $7(\mathrm{Abs}(\%))$ in Table 5, the number " 3133 " should be changed to " 3.133 ".

The online version of the original article can be found at https://doi.org/10.1007/s10489-018-1230-2.

$\triangle$ Jun $\mathrm{Ou}$

ccscogh@hotmail.com

1 Faculty of Information Technology, Beijing University of Technology, Beijing 100124, People's Republic of China
(4) In the 3nd row of Column $8(\operatorname{Rel}(\%))$ in Table 6, the number " 52419 " should be changed to " 5.2419 ".

(5) In the 2 rd row of Column $8(\operatorname{Rel}(\%))$ in Table 8 , the number " 10625 " should be changed to " 1.0625 ".

(6) In Figs. 7, 9, 10 and 11, "Recognition Accuracy(\%)", should be changed to "Recognition Accuracy", and their abscissa coordinates, i.e., "Iteration", should be replaced with "Number of Iterations".

The original version has been corrected. 